\title{
Vision from the right stem
}

\author{
Graziella Pellegrini ${ }^{1}$, Paolo Rama ${ }^{2}$ and Michele De Luca ${ }^{1}$ \\ ${ }^{1}$ Centre for Regenerative Medicine "Stefano Ferrari", University of Modena and Reggio Emilia, Modena, Italy \\ ${ }^{2}$ San Raffaele Scientific Institute, Ophthalmology Unit, Milan, Italy
}

\begin{abstract}
Cultures of limbal cells are a safe and effective treatment for the destruction of the human cornea owing to chemical burns. The essential feature of the graft is the presence of an adequate number of stem cells, which can be determined by the expression of the p63 transcription factor. Here, we will discuss the general principles defining the rigorous criteria for graftable limbal cultures in light of their clinical performances. Such criteria might prove relevant to the future therapeutic use of any cultured cell type.
\end{abstract}

\section{Stem cells and epithelial grafts}

Cultures of autologous keratinocytes have long been used to prepare grafts that can permanently restore severe epithelial defects, such as massive skin and ocular burns [1]. There have also been failures of this technology, which can be attributed to the lack of criteria for the quality control of cultures, inappropriate media, the components and/or substrates used for the cultures, the inadequacy of the preparation of the wound bed and/or surgical procedures [1]. In studying the low success rates described in the literature, we found it impossible to distinguish the contributions of these variables to the clinical performances of the cultures. Rather, the regeneration of selfrenewing tissues requires specific stem cells [2-4]; therefore, an essential feature of any epithelial graft is adequate numbers of stem cells, which are instrumental for the renewal and restoration of squamous epithelia [1]. Only when this criterion is met is it possible to separately assess the clinical/surgical factors. Here, we describe criteria for estimating the numbers of stem cells contained in grafts generated by corneal stem cells, which can serve as a paradigm for other types of squamous epithelia. We analyze these criteria from a patient's perspective (i.e. based on clinical outcomes) to see whether these data can shed light on the nature of epithelial stem cells and help define requirements for epithelial cultures destined for clinical application.

\section{The corneal epithelium and its limbal precursor}

The human ocular surface is covered by corneal, limbal and conjunctival squamous epithelia (Figure 1a). The corneal epithelium is flattened, transparent and stratified; it contains a basal layer of cuboidal cells lying on the Bowman's membrane (see Glossary) of an avascular corneal stroma. Corneal renewal and repair are executed by stem cells located in the limbus, the narrow zone between the cornea and the bulbar conjunctiva [5] (Figure 1a,b). Relatively undifferentiated slow-cycling epithelial cells have been found in the limbal basal layer but not in the central cornea [6,7]. Cells migrate from the limbus towards a wounded cornea [8,9]; mathematical analyses of the maintenance of the corneal epithelial cell mass [10] and the mosaic analysis of stem cell function and corneal wound healing [11] support the hypothesis that the corneal epithelium is regenerated by limbal stem cells. In the absence of a functional limbus, repeated corneal wounds result in progressive vascularization and recurrent erosions of the cornea, confirming that the corneal epithelium per se has a limited regenerative capacity [12]. During normal homeostasis, the murine central corneal epithelium could be self-sustaining and limbal stem cells might be recruited only after corneal injury [13]. So far, however, human corneal stem cells have been exclusively found in the limbus [13,14].

The transparency of the cornea, which depends on stromal avascularity and epithelial integrity, is essential to visual acuity. Ocular chemical or thermal burns can destroy the limbus, causing limbal stem cell deficiency (LSCD). Because the corneal epithelium can no longer be formed, the cornea acquires an epithelium by the invasion of bulbar conjunctival cells that originate beyond the destroyed limbus. This process leads to neovascularization, chronic inflammation, severe symptoms, corneal opacity and the loss of vision. Allogeneic corneal transplantation (keratoplasty; see Glossary), aimed at replacing the scarred corneal stroma and the inner endothelium, is not in itself a successful treatment. Although it temporarily removes the opacity, the conjunctival cells will resurface

\section{Glossary}

Bowman's membrane: a smooth layer located between the epithelium and the stroma in the cornea. It is composed of collagen fibers and helps the cornea maintain its shape.

Palisades of Vogt. crests of epithelium folds that run radially towards the cornea, at the limbus, from the bulbar conjunctiva. They are considered the "niche" containing limbal stem cells.

Keratoplasty: allogeneic corneal transplantation, also known as keratoplasty, is a surgical procedure where a damaged or diseased cornea is replaced by donated corneal tissue (the graft) in its entirety (penetrating keratoplasty) or in part (lamellar keratoplasty). It is aimed at replacing the corneal stroma and the inner endothelium, but not the corneal epithelium.

Amblyopia: a disorder of the visual system that is characterized by poor or indistinct vision in an eye that is otherwise physically normal or out of proportion with associated structural abnormalities.

Hypospadia: a congenital penile pathology characterized by an insufficient urethral development. Posterior hypospadia is the most severe form of this disease: the external urethral meatus is located at the base of the penis or in the scrotum. Junctional Epidermolysis Bullosa (JEB): Epidermolysis Bullosa is a family of inherited skin adhesion disorders causing the disruption of the epidermaldermal junction. JEB is a devastating (often lethal) form of the disease, where tissue separation occurs within the lamina lucida of the basement membrane. JEB results from mutations in genes encoding laminin 5 , the $\alpha 6 \beta 4$ integrin and collagen type XVII.

Corresponding author: De Luca, M. (michele.deluca@unimore.it). 


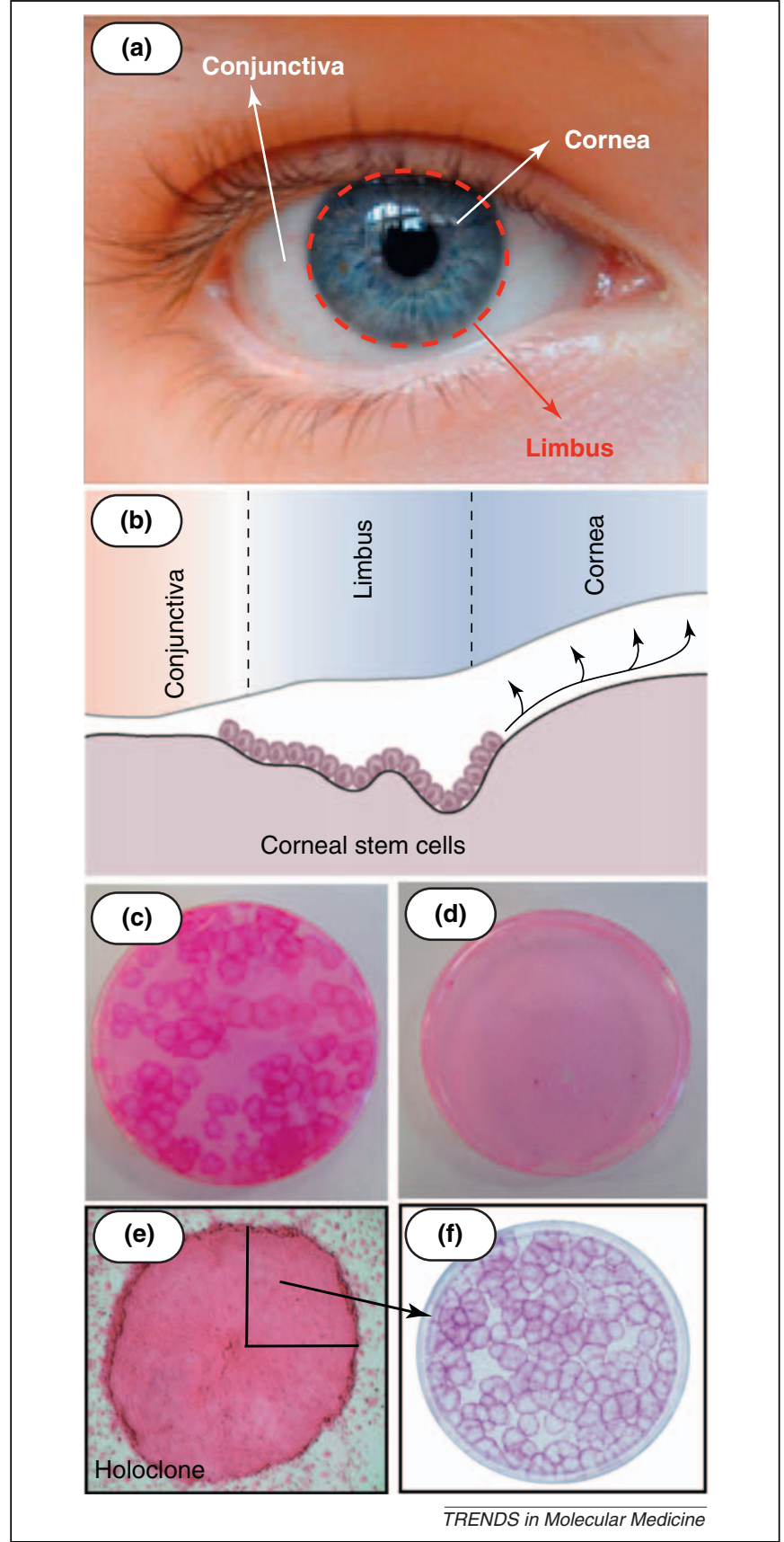

Figure 1. Corneal stem cells.

(a) The location of the limbus on the ocular surface. (b) Diagram showing the location of the limbus as well as the migration and differentiation of the limbal stem cells to form the corneal epithelium. (c and d) Colony-forming cells can be obtained from the limbus (c) but not the central cornea (d). The assay was performed as described [14]. (e) A holoclone isolated from a primary limbal culture. (f) Daughter colonies obtained from a quarter of a colony generated by a single holoclone. Limbal cultures and clonal analysis were performed as previously described [14].

the cornea. The only way to prevent this invasion is to restore the limbus, which can been attained in unilateral LSCD through the grafting of limbal fragments from the uninjured eye [15].

\section{Corneal regeneration by cultures of limbal stem cells}

The discovery that limbal cultures [16] contain stem cells [14] inspired the first therapeutic use of such cultures for the regeneration of a functional corneal epithelium [17]. Trypsinized cells of a single limbal biopsy of $1-2 \mathrm{~mm}^{2}$ are

\section{Box 1. Technical considerations}

- Serum selection: the batch of FCS should be tested for the preservation of clonogenic cells, the presence of aborted colonies and the maintenance of p $63^{\text {bright }}$ cells, using a reference batch of FCS. An appropriately selected FCS would assure the reproducibility of the cultures, eliminate the variability of autologous serum and allow rigorous in-process quality controls.

- Stem cell number: the number of stem cells present in a limbal biopsy is in the order of hundreds. Number increases during the primary culture because of the amplification of the original stem cell population. Amplification is the basis for the clinical success of the grafts. The same holds true for the cultivation of epidermal stem cells. The entire epidermis of a human being can be permanently regenerated (decades) from a small $\left(2 \mathrm{~cm}^{2}\right)$ skin biopsy.

- Feeder layer: a clone of 3T3 cells produces an identical feeder layer for all cultures, which assures a high reproducibility of the cultures, eliminates the variability of autologous fibroblasts (or other autologous cells) and allows rigorous in-process quality controls. It is safer than other allogeneic human mesenchymal cells because the 3 Т 3 Master and Working Cell Banks can be produced under rigorous GMP conditions, eliminating the risk of transmissible disease.

- p63 detection: $\Delta N p 63 \alpha$ is by far the most abundant p63 isoform contained in limbal holoclones. The $\beta$ and $\gamma$ isoforms are expressed at much lower levels and mostly in transient amplifying cells. Automated immunofluorescence detects only cells expressing very high levels of p63, thereby $\Delta N p 63 \alpha$, which can be equally detected by the pan-p63 4A4 mAb and the p63 $\alpha$-specific antiserum. Indeed, the entire procedure has been validated using the $4 \mathrm{~A} 4$ antibody and related to the number of holoclones, detected by rigorous clonal analysis.

cultivated on a feeder layer of lethally irradiated clinical grade [(GMP (good manufacturing practice)-certified] 3T3J2 cells in the presence of an appropriately selected fetal calf serum (FCS; Box 1). The subconfluent primary culture is trypsinized, a portion of the cells is transferred to a secondary culture on a fibrin disc of $3 \mathrm{~cm}$ diameter (on 3T3J2 feeder cells) [18-20] and the remainder are cryopreserved. When the secondary culture is confluent it is grafted over the corneal and limbal region of the injured eye, whose receiving bed had previously been surgically prepared by the removal of the abnormal epithelium and fibrovascular tissue [18]. This procedure reduces the loss of uninjured limbus and offers a therapeutic option to patients with severe bilateral corneal epithelial loss.

Approximately 15 years have passed since the first application of cultured limbal grafts to regenerate a corneal epithelium of patients suffering from LSCD [17]. Subsequent related studies (reviewed in [1,21-25]) have been limited by comparatively small numbers of cases, heterogeneous etiology, limited follow-up (often less than one year) and heterogeneous sources of transplant and cell culture. Cells have been cultured on a variety of substrates and culture media in the presence or absence of feeder cells or FCS. Patients have been treated with autologous or allogeneic limbal or oral cells. In these studies, stringent quality criteria for limbal cultures were not adopted. Although the procedures were often successful, it is not surprising that failures were reported and data were difficult to interpret.

\section{Learning from clinical outcomes}

Long-term clinical results in a homogeneous group of 112 patients presenting with unilateral $(87.5 \%)$ or bilateral 
Table 1. Clinical outcome of autologous limbal cultures ${ }^{*}$

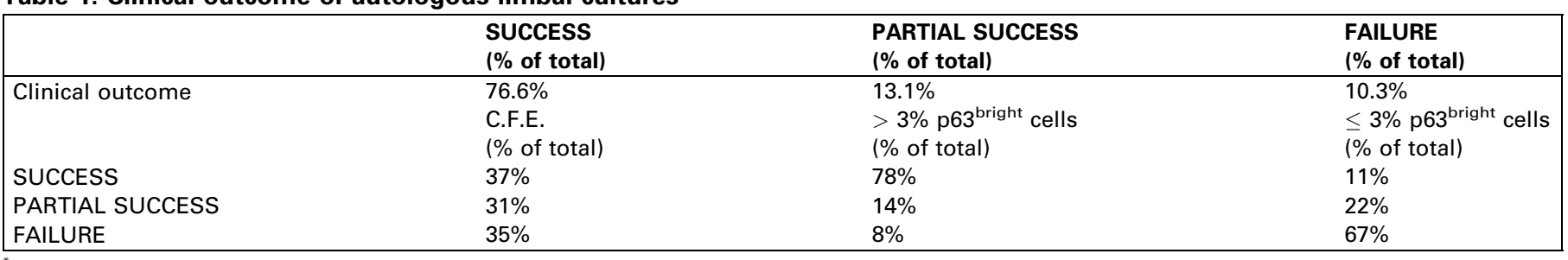

*Colony Forming Efficiency (C.F.E.) and quantitative immunodetection of p63 were performed on sub-confluent primary cultures used for the preparation of the grafts.

(12.5\%) burn-dependent LSCD and treated with cultures of autologous limbal cells have been reported [26]. The majority of eyes had undergone previous (sometimes multiple) surgeries, including keratoplasty, all of which failed because of a lack of residual limbal stem cells to regenerate the corneal epithelium. Preoperative best-corrected visual acuity was less than 0.1 (i.e. light perception, hand movements, counting fingers) in $88.5 \%$ of the patients [26]. Grafts of limbal cultures permanently restored a transparent, renewing and functional corneal epithelium, and thereby visual acuity recovered in $76.6 \%$ of the eyes [26] (Table 1). All failures occurred within 3-12 months of grafting.

All of the eyes restored at one year invariably remained stable over time (up to 10 years) and showed a transparent avascular cornea covered by a normal looking epithelium [26]. Limbal cultures sufficed to restore normal vision in patients with an undamaged corneal stroma (Figure 2a). To improve the visual acuity of eyes with stromal damage, approximately $40 \%$ of the patients underwent keratoplasty to remove the scarred stroma 12 to 24 months after grafting (Figure $2 \mathrm{~b}$ ). Keratoplasty is a formidable challenge to the engrafted stem cells, which for a second time generated the corneal epithelium necessary to permanently resurface the underlying donor stroma (Figure $2 \mathrm{~b}$ ).

Of note, clinical success was not associated with the total number of clonogenic cells; rather it correlated with the number of stem cells detected as holoclones, a specific type of clonogenic keratinocyte [26].

\section{Determination of stem cell numbers by clonal type}

Human epithelial cells can be studied at the clonal level. Clonogenic keratinocytes generate holoclones, meroclones and paraclones, which have different capacities for multiplication [14,27]. The holoclone-forming cell is the smallest colony-founding keratinocyte $[27,28]$, generates all the epithelial lineages of the tissue of origin [29] and has self-renewal ability [1,30], telomerase activity [31] and an impressive proliferative capacity [14]. The progression of clonal type follows holoclone $\rightarrow$ meroclone $\rightarrow$ paraclone, the latter having properties expected of transient amplifying progenitors because it has limited proliferative capacity. Meroclones have an intermediate proliferative potential and are a reservoir of paraclones [14,27].

Holoclones represent approximately $1-5 \%$ of clonogenic keratinocytes, the vast majority of which generate meroclones and paraclones. Although paraclones can be visually distinguished from the other clonal types, it is not possible to distinguish holoclones from meroclones solely based on the visual inspection of the colonies or measurement of the colony size. Meroclone-forming cells might even generate larger colonies than holoclone-forming cells in short-term clonogenic assays. However, clonogenic ability and regenerative potential are two different concepts. The former indicates the capacity of a cell to found a colony, whereas the latter deals with the long-term production of progenitors and self-renewal. Regenerative properties are possessed only by holoclones, which are the stem cells of

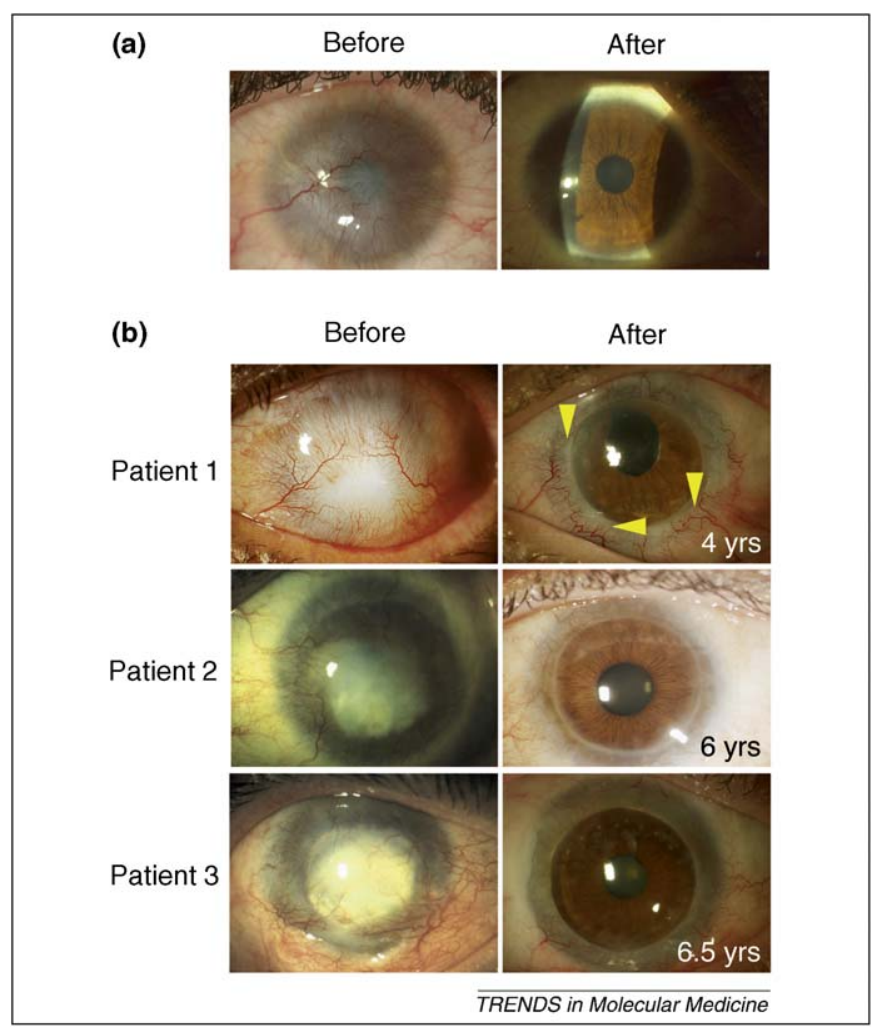

Figure 2. Regeneration of a functional corneal epithelium and restoration of visual acuity.

(a) The left eye of a patient who had total LSCD owing to an acid burn (left). His visual acuity was reduced to counting fingers. A graft of an autologous limbal culture was sufficient to regenerate a functional corneal epithelium (right) and to restore normal vision (visual acuity, 0.7 ), because the eye had no stromal scarring. (b) The eyes of three patients damaged by alkali burns and treated with unsuccessful surgery 3,13 and 30 years before admission, respectively. All three eyes had total LSCD, complete corneal opacification and stromal scarring (left). Vision was reduced to perceiving hand movements (in patients 1 and 3 ) or counting fingers (in patient 2). In all three patients, autologous limbal stem cell cultures successfully regenerated the functional corneal epithelium. To remove stromal scarring and improve visual acuity after grafting, the patients underwent penetrating keratoplasty. In all three eyes, the engrafted limbal stem cells resurfaced the donor stroma. At the last follow-up visits (at 4, 6 and 6.5 years, respectively), all eyes were covered by a stable corneal epithelium (right). Keratoplasty completely restored visual acuity in patients 1 and $2(0.8$ and 0.9 , respectively). The visual acuity of patient 3 increased to only 0.3 because of concomitant amblyopia (see Glossary). In patient 1, the follow-up image shows that the conjunctival vessels stop at the conjunctival-corneal boundary (arrowheads); they do not invade the restored corneal surface. Reproduced with permission from [26]. 
virtually all human squamous epithelia [1]. Indeed, keratinocyte cultures containing holoclones can permanently restore massive epithelial defects, including skin burns covering over $90 \%$ of the body surface and a complete lack of urethral epithelium in congenital posterior hypospadias [17-20,32,33] (see Glossary). Holoclones can be retrieved from the regenerated epidermis years after engraftment, showing that the stem cells did not lose their self-renewal capability during the cultivation process [1]. Finally, a defined number of genetically corrected holoclones can regenerate normal epidermises in patients with genetic skin adhesion disorders, such as Junctional Epidermolysis Bullosa [34] (see Glossary).

Clonogenic keratinocytes able to multiply are located in the limbus (Figure 1c) but not in the central part of the human cornea (Figure 1d) [14]. As mentioned, only $1-5 \%$ of the limbal clonogenic cells generate holoclones (Figure 1e,f), which are not contained in the central cornea $[13,14]$ Human corneal epithelia are thereby formed by limbal-derived progenitors. Cells in the peripheral cornea (very close to the limbus) have a residual, limited proliferative capacity, whereas those in the paracentral and central cornea generate only aborted colonies $[14,30]$ (Figure 1d).

\section{p63 as a stem cell determinant}

Some debate exists as to which molecule(s) should be considered definitive limbal stem cell markers (reviewed in $[25,35])$. Limbal-associated polypeptides $(\alpha 9$ and $\beta 1$ integrins, nerve growth factor receptors, CD133, $\alpha$-enolase and the metabolic enzymes, vimentin and K19) are expressed by the majority of limbal basal cells as well as by corneal cells and transient amplifying progenitors. The ATP-binding cassette transporter protein (ABCG2) marks a small percentage of limbal cells and is not present in the corneal epithelium. Notch 1 is detected in patches of ABCG2-positive limbal cells (reviewed in $[25,35]$ ). Although the expression of some of these proteins is consistent with limbal stem cells, their role in regulating stem cell function is poorly defined. Furthermore, only p63, the CCAAT enhancer-binding protein (C/EBP)- $\delta$ and Bmi1 transcription factors have been formally detected in human holoclones identified by rigorous clonal analysis [30].

TP63, the gene encoding p63, generates six isoforms [36]. TAp63 is generated by an upstream promoter, whereas $\Delta \mathrm{Np} 63$ is produced from a downstream promoter and isoforms derived from this transcript lack the transactivation domain. For both transcripts, alternative splicing gives rise to $\alpha, \beta$ and $\gamma$ isoforms [36]. p63 is essential for the generation and regeneration of squamous epithelia $[37,38]$. but its function in the development of these epithelia is still the subject of controversy [39,40]. There is a good deal of evidence supporting the hypothesis that p63 promotes the maintenance of epithelial stem cells and is a determinant of their proliferative potential [37,41-44]. In newborn $p 63^{-1-}$ mice, terminally differentiated suprabasal keratinocytes are present but there is no detectable proliferative basal layer containing the stem cell population necessary to sustain the epithelium [37]. p63 is strongly expressed in epidermal and limbal holoclones [42]. Epithelial stem cells lacking p63 undergo a premature proliferative rundown [43]. p63 maintains adult epithelial stem cells by regulating cellular senescence and genomic stability, thereby preventing premature tissue aging [44].

Human limbal and corneal keratinocytes might contain all $\Delta \mathrm{N}$ isoforms, but $\Delta \mathrm{Np} 63 \alpha$ is by far the most abundant. In the uninjured surface of the eye, $\Delta \mathrm{Np} 63 \alpha$ is in the limbus (in patches of basal cells) but absent from the central cornea $[42,45] . \Delta \mathrm{Np} 63 \beta$ and $\Delta \mathrm{Np} 63 \gamma$ appear upon wounding and correlate with corneal differentiation. $\Delta \mathrm{Np} 63 \alpha$ is expressed at high levels in holoclones, at low levels in meroclones but is undetectable in paraclones $[42,45]$. The transition from holoclone to paraclone is thereby accompanied by the progressive disappearance of $\Delta \mathrm{Np} 63 \alpha$ and relative enrichment of $\Delta \mathrm{Np} 63 \beta$ and $\Delta \mathrm{Np} 63 \gamma[42,45]$. The coexpression of $\mathrm{C} / \mathrm{EBP} \delta, \mathrm{Bmi1}$ and $\Delta \mathrm{Np} 63 \alpha$ identifies mitotically quiescent limbal cells located in the Palisades of Vogt (see Glossary) and thereby determines part of the genetic program maintaining human limbal stem cell identity. Accordingly, holoclones contain $\mathrm{C} / \mathrm{EBP} \delta$ and Bmi1, both of which are undetectable in meroclones and paraclones. $\Delta \mathrm{Np} 63 \alpha$ sustains the proliferative potential of limbal stem cells, and C/EBP $\delta$ (and probably Bmi1) regulates their self-renewal and mitotic rate, whereas $\Delta \mathrm{Np} 63 \beta$ and $\Delta \mathrm{Np} 63 \gamma$ are associated with corneal terminal differentiation [30].

The abundance of $\Delta \mathrm{Np} 63 \alpha$ (hereafter referred to as p63) in holoclones made it possible to prospectively evaluate the number of stem cells in a limbal graft without the need for clonal analysis. The frequency of p63-positive cells can be evaluated by the computerized analysis of the intensity of the staining of single cells, as assessed by automated quantitative immunocytochemistry, using as a reference a limbal strain that contains a known percentage of holoclones $[1,46]$. Holoclone content, long-term proliferative capacity and the expression of high levels of p63 correlated well in both mass and clonal cultures. The quantitative immunodetection of $\mathrm{p} 63^{\text {bright }}$ holoclones is straightforward and can be performed before grafting [46].

\section{The role of $p 63^{\text {bright }}$ holoclones in permanent corneal regeneration}

The clinical success of engrafted limbal cultures was strikingly correlated to the percentage of stem cells detected as p63 $3^{\text {bright }}$ holoclones [26]. Cultures containing more than $3.0 \%$ of $\mathrm{p} 63^{\text {bright }}$ holoclones were successful in almost $80 \%$ of patients. By contrast, cultures containing $3.0 \%$ or less of such cells were associated with successful transplant in only $11 \%$ of patients [26] (Table 1). This indicates that at least 3000 stem cells are required to achieve clinical success: a primary limbal culture contains a minimum of $3 \times 10^{5}$ cells, about $30 \%$ of which are clonogenic; thus, $3 \%$ of these clonogenic cells correspond to approximately 3000 stem cells (Box 1). Because holoclones represent a tiny percentage $(\sim 1 \%)$ of the total cells grafted on the patient, corneal regeneration achieved in these patients cannot be ascribed to a nonspecific stimulatory effect of epithelial cultures (or surgical manipulation) on spared resident limbal cells (should such be present).

Of note, clinical data formally prove that a colony-forming efficiency assay (which has been used as a stem cell assay) is not sufficient to evaluate the number of stem cells 
and predict the performance of the graft. Indeed, the colony-forming efficiency of successful and unsuccessful cultures was virtually identical (Table 1). This is highly relevant because epithelial grafts containing holoclones are visually undistinguishable from those devoid of them.

Kaplan-Meyer estimates of the survival of the cultures showed that all failures occurred within the first year of grafting, whereas successful cultures remained stable for up to 10 years [26]. Grafts devoid (or containing an insufficient number) of stem cells (p63 ${ }^{\text {bright }}$ holoclones) might thereby account not only for some of the early failures but also for some of the reported corneal instability leading to corneal reopacification in the long-term [47]. This concept also holds true for epidermal regeneration in fullthickness skin burns by cultures of autologous epidermal keratinocytes. The poor quality of the epidermises regenerated on some patients and/or the commonly observed disappearance of the epidermis after an initial take can be attributed, at least in part, to the deterioration of the stem cells during the cultivation [1]. It is common knowledge among plastic surgeons that poor quality epidermal cultures either do not engraft or last only a few weeks after transplantation. Similarly, corneal instability, presumably arising from a poor quality limbal culture, might appear months after grafting [47]. This might reflect the fact that the human epidermis is renewed monthly, whereas the ocular epithelium is renewed every 9-12 months. Accordingly, ocular holoclones have a lower proliferative potential (90-100 cell doublings) than epidermal holoclones (140-180 cell doublings), suggesting that holoclones can adjust their proliferative potential according to the tissue of origin [14].

\section{Definition of quality criteria}

Although holoclones as a determinant of the regenerative capacity of any keratinocyte culture have been recognized [1], the data tell us that a discrete number of stem cells is required to achieve clinical success [26]. Stringent quality criteria should be adopted for the keratinocyte cultivation process. This does not mean that the cultures should contain a well-organized stratified epithelium, but rather that they must contain a sufficient number of stem cells essential for long-term epithelial renewal.

During the past few years, new culture technologies have been proposed for limbal cultures, envisaging new culture media and/or the cultivation of limbal cells onto different carriers as well as with cells in suspension (reviewed in [1,21-25]). The maintenance of holoclones by rigorous clonal and/or molecular analyses has never been demonstrated in these conditions. Sometimes, the methods used to prepare cultures do not favor the retention of stem cells. One example is the use of explant cultures, which have been shown to determine a loss of stem cells [48]. Another is the use of "air-lift cultures," which promote terminal differentiation but not the retention of stem cells [49]. Irreversible clonal evolution occurs during serial keratinocyte cultivation. Incorrect (or nonvalidated) culture conditions can accelerate this clonal conversion, thereby causing a rapid disappearance of stem cells (G. Pellegrini et al., unpublished) rendering cultured autografts useless. In such cases, failures are inevitable and will entail not only the suffering of the patients (and possible loss of life in the case of massive skin burns), but also confusion as to what results should be expected.

A rigorous clonal analysis (in which single cell-derived colonies are analyzed and scored as holoclones after subcultivation) $[14,27]$ and/or the evaluation of the number of cell doublings generated during the serial cultivation of cells [14] should, therefore, be adopted to validate a newly proposed limbal culture system. However, these assays would be cumbersome as a standard routine test for the quality of cultured grafts. The number of aborted colonies (paraclones) is inversely related to the number of holoclones and is easier to score. The proportion of aborted colonies rises slightly during the two stages of cultivation required to prepare the graft but the mean value does not exceed $10 \%$ of the total. Autologous cultures of this quality are acceptable for grafting [1].

Data from a long-term clinical study have suggested that the identification of holoclones by the immunodetection of p63 is another important and simple means of determining the presence of an adequate number of stem cells in a cultured keratinocyte graft [26]. This does not mean that any positivity for $\mathrm{p} 63$ should be considered a marker for limbal stem cells. The strict correlation of clinical performance with the level of expression of p63 indicates that $\mathrm{p} 63^{\text {bright }}$ cells should first be validated by a quantitative assay and correlated with the number of holoclone-forming cells contained in the culture [46].

\section{The role of the microenvironment}

The presence of a defined amount of stem cells in the graft is not the only factor determining clinical success. Chemical burns might damage the eyelids, the conjunctiva and the lachrymal system. During deterioration, the ocular surface is chronically inflamed, which might alter the microenvironment, and in turn, hamper the engraftment of cultured stem cells [26] (Box 2). We have no sense for the number of stem cells that can engraft on the wound bed; it is possible that the failed transplants that contained an appropriate number of $\mathrm{p} 63^{\text {bright }}$ cells lost stem cells during engraftment owing to a hostile in vivo microenvironment. The proper selection of patients, the definition of stringent inclusion and exclusion criteria and the proper preparation of the damaged ocular surface during the weeks/months

\section{Box 2. Failures in the presence of $p 63^{\text {bright }}$ cells}

1. Uncontrolled inflammation

2. Tear film deficiency

3. Global severity of the ocular damage

4. Drug toxicity

Failures associated with cultures containing an appropriate number of stem cells strongly suggest that stem cells might be lost during engraftment because of a hostile in vivo microenvironment. Thus, the following exclusion criteria should be adopted:

1. Active ocular inflammation

2. Eyelid malposition

3. Conjunctival scarring with fornix shortening

4. Severe tear film deficiency

5. Corneal and conjunctival anesthesia 
preceding the grafting procedure might also be crucial to success [26] (Box 2).

\section{Implications for regulation}

According to the new regulations on advanced therapies, autologous cultures of epithelial cells must adhere to GMP. Cultured cells, however, are not defined as chemical compounds and pose an array of additional, perhaps more complicated issues. Regulations on advanced therapies should take into consideration the biology of different cells and should, therefore, be specific to a particular cell type, a particular modality of action and a particular method of delivery (i.e. autologous versus allogeneic tissue; minimal processing versus extensive manipulation; homologous versus nonhomologous use). Given the inherent variability of cells and the stringent requirements of establishing manufacturing uniformity, GMP for cell-based medicine will have to be flexible and tailored to the cell type [50].

For instance, one of the most important GMP-related issues is the need for animal components in cultivation. The maintenance of keratinocyte stem cells in culture requires an appropriate feeder layer of lethally irradiated 3T3 cells - the 3T3-J2 clone - and an appropriately selected FCS [1]. To our knowledge, no other method assures the preservation of holoclones as detected by rigorous clonal analysis. The 3T3-J2 clone and FCS have been used since 1979 in many countries (including the US, Italy, France, the UK, Germany, Switzerland, Japan and others) to culture epithelial cells (such as epidermal, limbal, urethral and oral keratinocytes) that have been transplanted on thousands of patients with severe epithelial defects, such as full-thickness burns covering up to $95 \%$ of the body surface or total corneal destruction [1]. In the past 30 years, no adverse effects derived from the use of a clinical grade, GMP-certified 3T3 Master Cell Bank have been reported. The use of such feeder cells has been authorized for clinical use by regulatory authorities in Italy, the US, Japan and South Korea. At variance with autologous fibroblasts, a feeder layer prepared from a highly standardized clone of 3T3 cells allows reproducibility in the cultivation process and the development of in-process controls with defined specification limits. Such criteria should prevent potential efficacy problems or unexpected adverse events derived from the use of a nonstandardized feeder layers.

The use of animal components is generally disfavored by (some) regulatory authorities, although the ISSCR Guidelines on Clinical Translation of Stem Cells (www.isscr.org/ clinical_trans) states that "inclusion of animal materials in the cell manufacturing process does not preclude human use, as stipulated in existing guidelines for medicinal products," provided that appropriate controls and certifications have been produced. It is important that regulations do not hinder the field while providing adequate protection for patients. In other words, GMP should not hamper the development of highly efficacious cultures and a careful assessment of risk/benefit should be made.

\section{Concluding remarks}

Patients with disabling diseases have contributed greatly to our understanding of stem cells. The interface between basic science and applicative cell therapy will certainly provide insights for all investigators operating in the eventful field of translational medicine, as it did for epithelial stem cell-based regenerative medicine. The difficulties encountered in keratinocyte therapy have been great but much progress has been made even if this progress is yet to be assimilated and applied by scientists and physicians. Therapy with limbal stem cells is ready for widespread use because the necessary criteria for graftable cultures and for their surgical use are now well understood. Therefore, we emphasize the importance of a discipline for defining the suitability and quality of cultured epithelial grafts, which are relevant to the future use of any cultured cell type for therapeutic purposes.

\section{Acknowledgements}

This work was supported by grants from the VII Framework Programme, OPTISTEM (HEALTH-F5-2009-223098) and the Regione Emilia Romagna (area 1b "Medicina Rigenerativa"). We regret not being able to cite all papers describing the clinical application of cultured limbal cells because of editorial space limitations. These papers are cited in $[1,22-25,35,47]$.

\section{References}

1 De Luca, M. et al. (2006) Regeneration of squamous epithelia from stem cells of cultured grafts. Regenerative Med. 1, 45-57

2 Blanpain, C. et al. (2007) Epithelial stem cells: turning over new leaves. Cell 128, 445-458

3 Blanpain, C. and Fuchs, E. (2009) Epidermal homeostasis: a balancing act of stem cells in the skin. Nat. Rev. Mol. Cell Biol. 10, 207-217

4 Watt, F.M. and Jensen, K.B. (2009) Epidermal stem cell diversity and quiescence. EMBO Mol. Med. 1, 260-267

5 Pellegrini, G. et al. (2009) Epithelial stem cells in corneal regeneration and epidermal gene therapy. J. Pathol. 217, 217-228

6 Schermer, A. et al. (1986) Differentiation-related expression of a major $64 \mathrm{~K}$ corneal keratin in vivo and in culture suggests limbal location of corneal epithelial stem cells. J. Cell Biol. 103, 49-62

7 Cotsarelis, G. et al. (1989) Existence of slow-cycling limbal epithelial basal cells that can be preferentially stimulated to proliferate: implications on epithelial stem cells. Cell 57, 201-209

8 Lehrer, M.S. et al. (1998) Strategies of epithelial repair: modulation of stem cell and transit amplifying cell proliferation. J. Cell Sci. 111 (Pt 19), 2867-2875

9 Nagasaki, T. and Zhao, J. (2003) Centripetal movement of corneal epithelial cells in the normal adult mouse. Invest. Ophthalmol. Vis. Sci. $44,558-566$

10 Sharma, A. and Coles, W.H. (1989) Kinetics of corneal epithelial maintenance and graft loss. A population balance model. Invest. Ophthalmol. Vis. Sci. 30, 1962-1971

11 Mort, R.L. et al. (2009) Mosaic analysis of stem cell function and wound healing in the mouse corneal epithelium. BMC Dev. Biol. 9, 4

12 Huang, A.J. and Tseng, S.C. (1991) Corneal epithelial wound healing in the absence of limbal epithelium. Invest. Ophthalmol. Vis. Sci. 32, 96-105

13 Majo, F. et al. (2008) Oligopotent stem cells are distributed throughout the mammalian ocular surface. Nature 456, 250-254

14 Pellegrini, G. et al. (1999) Location and clonal analysis of stem cells and their differentiated progeny in the human ocular surface. J. Cell Biol. $145,769-782$

15 Kenyon, K.R. and Tseng, S.C. (1989) Limbal autograft transplantation for ocular surface disorders. Ophthalmology 96, 709-722, discussion 722-703

16 Lindberg, K. et al. (1993) In vitro propagation of human ocular surface epithelial cells for transplantation. Invest. Ophthalmol. Vis. Sci. 34, 2672-2679

17 Pellegrini, G. et al. (1997) Long-term restoration of damaged corneal surfaces with autologous cultivated corneal epithelium. Lancet 349, 990-993

18 Rama, P. et al. (2001) Autologous fibrin-cultured limbal stem cells permanently restore the corneal surface of patients with total limbal stem cell deficiency. Transplantation 72, 1478-1485 
19 Pellegrini, G. et al. (1999) The control of epidermal stem cells (holoclones) in the treatment of massive full-thickness burns with autologous keratinocytes cultured on fibrin. Transplantation 68, 868-879

20 Ronfard, V. et al. (2000) Long-term regeneration of human epidermis on third degree burns transplanted with autologous cultured epithelium grown on a fibrin matrix. Transplantation 70, 1588-1598

21 Schwab, I.R. et al. (2000) Successful transplantation of bioengineered tissue replacements in patients with ocular surface disease(1). Am. J. Ophthalmol. 130, 543-544

22 Shortt, A.J. et al. (2007) Transplantation of ex vivo cultured limbal epithelial stem cells: a review of techniques and clinical results. Surv. Ophthalmol. 52, 483-502

23 Takacs, L. et al. (2009) Stem cells of the adult cornea: from cytometric markers to therapeutic applications. Cytometry A 75, 54-66

24 McIntosh Ambrose, W. et al. (2010) A tale of two tissues: stem cells in cartilage and corneal tissue engineering. Curr. Stem Cell Res. Ther. 5, $37-48$

25 Notara, M. et al. (2010) In sickness and in health: corneal epithelial stem cell biology, pathology and therapy. Exp. Eye Res. 90, 188-195

26 Rama, P. et al. (2010) Limbal stem-cell therapy and long-term corneal regeneration. N. Engl. J. Med. 363, 147-155

27 Barrandon, Y. and Green, H. (1987) Three clonal types of keratinocyte with different capacities for multiplication. Proc. Natl. Acad. Sci. U. S. A. 84, 2302-2306

28 Barrandon, Y. and Green, H. (1985) Cell size as a determinant of the clone-forming ability of human keratinocytes. Proc. Natl. Acad. Sci. U. S. A. $82,5390-5394$

29 Claudinot, S. et al. (2005) Long-term renewal of hair follicles from clonogenic multipotent stem cells. Proc. Natl. Acad. Sci. U. S. A. 102, 14677-14682

30 Barbaro, V. et al. (2007) C/EBPdelta regulates cell cycle and selfrenewal of human limbal stem cells. J. Cell Biol. 177, 1037-1049

31 Dellambra, E. et al. (2000) Downregulation of 14-3-3sigma prevents clonal evolution and leads to immortalization of primary human keratinocytes. J. Cell Biol. 149, 1117-1130

32 Gallico, G.G., III et al. (1984) Permanent coverage of large burn wounds with autologous cultured human epithelium. N. Engl. J. Med. 311, $448-451$

33 Romagnoli, G. et al. (1990) Treatment of posterior hypospadias by the autologous graft of cultured urethral epithelium. N. Engl. J. Med. 323, $527-530$
34 Mavilio, F. et al. (2006) Correction of junctional epidermolysis bullosa by transplantation of genetically modified epidermal stem cells. Nat. Med. 12, 1397-1402

35 Secker, G.A. and Daniels, J.T. (2008) Corneal epithelial stem cells: deficiency and regulation. Stem Cell Rev. 4, 159-168

36 Yang, A. et al. (1998) p63, a p53 homolog at 3q27-29, encodes multiple products with transactivating, death-inducing, and dominant-negative activities. Mol. Cell 2, 305-316

37 Yang, A. et al. (1999) p63 is essential for regenerative proliferation in limb, craniofacial and epithelial development. Nature 398, 714-718

38 Mills, A.A. et al. (1999) p63 is a p53 homologue required for limb and epidermal morphogenesis. Nature 398, 708-713

39 Koster, M.I. et al. (2004) p63 is the molecular switch for initiation of an epithelial stratification program. Genes Dev. 18, 126-131

40 Koster, M.I. et al. (2005) P63 deficiency: a failure of lineage commitment or stem cell maintenance? J. Investig. Dermatol. Symp. Proc. 10, 118-123

41 Parsa, R. et al. (1999) Association of $\mathrm{p} 63$ with proliferative potential in normal and neoplastic human keratinocytes. J. Invest. Dermatol. 113, 1099-1105

42 Pellegrini, G. et al. (2001) p63 identifies keratinocyte stem cells. Proc. Natl. Acad. Sci. U. S. A. 98, 3156-3161

43 Senoo, M. et al. (2007) p63 Is essential for the proliferative potential of stem cells in stratified epithelia. Cell 129, 523-536

$44 \mathrm{Su}, \mathrm{X}$. et al. (2009) TAp63 prevents premature aging by promoting adult stem cell maintenance. Cell Stem Cell 5, 64-75

45 Di Iorio, E. et al. (2005) Isoforms of DeltaNp63 and the migration of ocular limbal cells in human corneal regeneration. Proc. Natl. Acad. Sci. U. S. A. 102, 9523-9528

46 Di Iorio, E. et al. (2006) Q-FIHC: quantification of fluorescence immunohistochemistry to analyse p63 isoforms and cell cycle phases in human limbal stem cells. Microsc. Res. Tech. 69, 983-991

47 Mason, C. and Manzotti, E. (2010) Regenerative medicine cell therapies: numbers of units manufactured and patients treated between 1988 and 2010. Regen. Med. 5, 307-313

$48 \mathrm{Li}$, W. et al. (2007) The fate of limbal epithelial progenitor cells during explant culture on intact amniotic membrane. Invest. Ophthalmol. Vis. Sci. 48, 605-613

$49 \mathrm{Li}$, W. et al. (2008) Air exposure induced squamous metaplasia of human limbal epithelium. Invest. Ophthalmol. Vis. Sci. 49, 154-162

50 McMahon, A.D. et al. (2009) The unintended consequences of clinical trials regulations. PLoS Med. 3, e1000131 